[Article]

\title{
甲醇芳构化中催化剂酸性对脱烷基、烷基化和异构化反应的影响
}

\author{
张金贵 $^{1}$ 骞伟中 ${ }^{1, *}$ 汤效平 ${ }^{1,2}$ 沈 葵 ${ }^{1}$ 王 䑣 ${ }^{1,2}$ 黄晓凡 ${ }^{2}$ 魏 飞 ${ }^{1}$ \\ ('清华大学化工系, 北京市绿色反应工程与工艺重点实验室, 北京 100084; \\ 2华电煤业集团有限公司煤化工事业部, 北京 100031)
}

\begin{abstract}
摘要: 在 Zn/P/ZSM-5 催化剂上研究了甲醇、二甲苯、甲苯和甲醇等不同进料下芳烃产品分布随反应积碳的变 化, 发现催化剂积碳对芳构化反应、脱烷基反应和烷基化反应的影响不同. 在不同硅铝摩尔比(Si/Al 比)和 Zn 负 载量的Zn/P/ZSM-5 催化剂上进行甲醇转化, 考察催化剂酸性位点强度、密度和类型与芳烃收率和产品分布之 间的关系, 发现当强酸位点酸密度下降时, 脱烷基反应最先被抑制, 其次是芳构化反应和异构化反应, 而烷基化 反应却不受影响. 在 Si/Al 比为 14, 3\% (w) Zn 负载量的 Zn/P/ZSM-5 催化剂上可得到 75\%左右的芳烃收率, 二 甲苯收率在 $35 \%$ 左右, 具有重要的工业应用前景.
\end{abstract}

关键词：甲醇；ZSM-5分子篎；芳构化；脱烷基；烷基化；异构化

中图分类号: 0643

\section{Influence of Catalyst Acidity on Dealkylation, Isomerization and Alkylation in MTA Process}

\author{
ZHANG Jin-Gui ${ }^{1} \quad$ QIAN Wei-Zhong ${ }^{1, *} \quad$ TANG Xiao-Ping ${ }^{1,2} \quad$ SHEN Kui ${ }^{1} \quad$ WANG Tong ${ }^{1,2}$ \\ HUANG Xiao-Fan ${ }^{2}$ WEI Fei ${ }^{1}$ \\ ('Beijing Key Laboratory of Green Reaction Engineering \& Technology, Department of Chemical Engineering, \\ Tsinghua University, Beijing 100084, P. R. China; ${ }^{2}$ Coal Chemical Industry Division, Huadian Coal Industry \\ Group Co. Ltd., Beijing 100031, P. R. China)
}

\begin{abstract}
Relationship between aromatics distribution, in the process of methanol to aromatics (MTA), and the conversion of methanol and the catalyst acidity was investigated over a series of Zn/P/ZSM- 5 catalysts with different Si/Al molar ratios and zinc loading. To understand the contribution of aromatization, isomerization, dealkylation and alkylation reactivity of the catalyst to the aromatics distribution, coke deposition degree of Zn/P/ZSM- 5 catalyst was tailored as using different feedstocks including methanol, xylene or the mixture of methanol and toluene. With the coke deposition, the amount of different types of acidic sites of catalyst varied significantly, characterized by $\mathrm{NH}_{3}$-temperature programmed desorption $\left(\mathrm{NH}_{3}-\mathrm{TPD}\right)$ and pyridine-infrared methods. Aromatization, dealkylation, alkylation, and isomerization showed sensitivity to a reduction in the density of strongly acidic sites. Dealkylation reaction was preferentially inhibited just by slightly decreasing the density of strong acid sites. However, aromatization and isomerization reaction were inhibited only when the density of strong acid sites was significantly decreased. In all cases, alkylation was found to be uninfluenced by acidic site density. A Zn/P/ZSM-5 catalyst with Si/Al molar ratio of 14 and $3 \%(w) \mathrm{Zn}$ loading exhibited aromatics yields of $75 \%$ and xylene yields of about $35 \%$, indicating potential for industrial application.
\end{abstract}

Key Words: Methanol; ZSM-5 zeolite; Aromatization; Dealkylation; Alkylation; Isomerization

Received: January 16, 2013; Revised: April 9, 2013; Published on Web: April 10, 2013.

"Corresponding author. Email: qianwz@mail.tsinghua.edu.cn; Tel: +86-10-62794133.

The project was supported by the National High Technology Research and Development Program of China (863) (2012AA051003).

国家高技术研究发展计划(863)(2012AA051003)资助项目

C. Editorial office of Acta Physico-Chimica Sinica 


\section{1 引 言}

由于石油资源日益紧张, 以来源于煤或天然气 的甲醇做原料, 利用催化转化的方法制备烃类尤其 是芳烃的技术路线越来越受到重视. ${ }^{1-3}$ 使用 ZSM-5 分子篮, 负载镓、铜、锌、银等金属, 甲醇一次通过转 化能够获得 50\%-70\%收率(碳基)的芳烃. ${ }^{4-10}$ 生成的 $\mathrm{C}_{2}-\mathrm{C}_{5}$ 轻烃也可以循环转化, 继续生成芳烃. 传统石 油路线可能得到几百种芳烃与非芳组分, 需要专 门、复杂、高能耗的芳烃抽提工段来分离. 而甲醇芳 构化一般只得到苯、甲苯、二甲苯、三甲苯和甲基萘 等十余种芳烃, 且甲基苯类芳烃的质量分数大于 $90 \%{ }^{9,10}$ 与传统路线相比, 该过程具有产品易分离、 能耗低的特点. 考虑到不同种类的芳烃产物(苯、甲 苯和二甲苯)用途不同, 理解相关催化剂上芳烃的生 成机理, 以及影响芳烃产品分布的关键因素, 以期 高选择性地制备某种芳烃, 是进一步研究的目标.

虽然甲醇制芳烃过程生产的芳烃种类较简单, 但目前学术界对不同芳烃的生成历程仍然没有统 一的认识. 前期研究中, 李文怀等 ${ }^{11}$ 使用 Zn/ZSM-5 分子篮, 在 $350{ }^{\circ} \mathrm{C}$ 转化甲醇, 发现可能存在着分子 量较大的醚类中间体, 在强 Brönsted (B)酸催化剂上 继续裂解为芳烃与烯烃, 但没有讨论芳烃分布的影 响因素. Kecskemeti 等 ${ }^{12}$ 比较了二甲醚与二乙醚的 芳构化特性, 二乙醚本身分子中就存在着烃基链, 而甲醇或二甲醚没有, 因此甲醇或二甲醚生成 $\mathrm{C}_{2}$ 以 上烃类物种的步骤可能影响到产物的分布. 当以二 甲醚为原料时, 产物中二甲苯的含量显著大于甲苯 与苯. 添加 $\mathrm{MoC}$ 后会促进三甲苯的生成, 但甲苯的 含量更低. Choudhary 等 ${ }^{13}$ 认为丙烯与丁烯芳构化会 率先生成二甲苯, 然后二甲苯再进行脱烷基或烷基 化反应生成别的甲基苯. Inoue 等 ${ }^{14}$ 利用 Ag/ZSM-5 在 $430-470{ }^{\circ} \mathrm{C}$ 转化甲醇, 得到了 $30 \%-40 \%$ 的二甲 苯与三甲苯, 而苯与甲苯的总量低于 $15 \%$. 而 Bjørgen 等 ${ }^{15}$ 利用瞬态 ${ }^{12} \mathrm{C} /{ }^{13} \mathrm{C}$ 同位素甲醇切换进料技 术, 分析了甲醇转化为芳烃与烯烃的特征, 提出可 能存在着双催化循环, 一个以三甲苯与甲苯为中间 体, 继而生成乙烯与芳烃; 另一个以高碳烷烃为中 间体, 继而生成丙烯与其它烷烃. Adebajo 等 ${ }^{16}$ 研究 不同温度下甲醇芳构化过程中苯的甲基化二次反 应, 发现在 $400{ }^{\circ} \mathrm{C}$ 以上该反应非常显著. 显然, 反应 温度、分子篮(不同 $\mathrm{Si} / \mathrm{Al}$ 比和不同酸性)、负载金属 和甲醇分压的不同, 导致了上述研究结果中芳烃产 品分布的差异.
本文认为, 如果简化甲醇生成芳烃的具体历 程, 则甲醇在 HZSM- 5 或改性 ZSM-5 分子篮上发生 转化时, 可依次生成二甲醚中间体、烃类中间体, 再 经聚合、环化和脱氢等步骤形成芳环. $3.1,17$ 当芳环生 成以后, 烷基化、脱烷基和异构化等反应的发生会 导致最终芳烃分布与初始芳烃分布的差异. ZSM-5 分子篎具有三维交叉孔道体系. 直孔道(沿[010] 晶 面、 $b$ 轴) 大小为 $0.54 \mathrm{~nm} \times 0.56 \mathrm{~nm}$, 正弦孔道(沿[100] 晶面、 $a$ 轴) 大小为 $0.51 \mathrm{~nm} \times 0.55 \mathrm{~nm} .{ }^{18}$ 孔道大小与二 甲苯分子大小相仿, 对直接生成苯、甲苯和对二甲 苯均不存在过渡态择形限制. 但是根据模拟计算, 甲醇芳构化中生成甲苯与对二甲苯在热力学上相 对有利. ${ }^{19}$ 分子篎的这种择形作用和反应热力学决 定了芳烃产品的初始分布. 而当催化剂孔内生成的 对二甲苯扩散到达表面后, 会发生异构化反应生成 间二甲苯与邻二甲苯, 最终三者组成为热力学平衡 组成. ${ }^{20-22}$ 同时甲苯和二甲苯可继续发生烷基化反 应, 分别生成二甲苯和三甲苯. 值得指出, 气相存在 的甲醇分子在催化剂表面吸附生成大量甲氧基与 甲基, 有利于甲醇与芳环的烷基化反应. ${ }^{23}$ 而且甲基 类芳烃还会在孔内和表面发生脱烷基化反应, 脱除 侧链烷基. 24,25 这些二次反应的发生决定了芳烃产品 的最终分布. 探究甲醇芳构化过程中烷基化、脱烷 基和异构化反应的影响因素, 尤其是这些反应和催 化剂酸性位点的关系, 有助于我们对甲醇制芳烃催 化剂的设计和改进, 以期在保持高芳烃收率的同时 得到理想的芳烃产品分布.

为了探究甲醇芳构化过程中影响主次反应和 芳烃分布的因素, 本文制备了一系列不同母体 $\mathrm{Si} / \mathrm{Al}$ 比和不同 $\mathrm{Zn}$ 负载量的 $\mathrm{Zn} / \mathrm{P} / \mathrm{ZSM}-5$ 催化剂, 在 $3 \%$ $\mathrm{Zn} / \mathrm{P} / \mathrm{ZSM}-5$ (14)催化剂上, 分别进行甲醇、二甲苯和 甲苯甲醇混合物的转化反应, 对应的主反应分别为 芳构化、脱烷基和异构化、烷基化反应. 芳构化、烷 基化和脱烷基反应随着催化剂反应积碳呈现出不 同的变化规律. 同时结合多种酸性表征手段, 探讨 不同 $\mathrm{Si} / \mathrm{Al}$ 比和 $\mathrm{Zn}$ 含量的催化剂上芳烃产品的分布 规律.

\section{2 实验部分}

不同 $\mathrm{Si} / \mathrm{Al}$ 比的氢型 ZSM-5 分子篮由南开催化 剂厂购得. 催化剂体相 $\mathrm{Si} / \mathrm{Al}$ 比由电感耦合等离子发 射光谱仪(ICP, 美国 VARIAN 公司 Vista-MPX型)测 定. 称取一定量的 ZSM-5 原粉在 $\left(\mathrm{NH}_{4}\right)_{2} \mathrm{HPO}_{4}$ 溶液中 
充分搅拌, 浸渍 $4 \mathrm{~h}, 110{ }^{\circ} \mathrm{C}$ 烘干并在 $500{ }^{\circ} \mathrm{C}$ 空气气 氛下焙烧. 磷改性量为 $3 \%$ (以 $\mathrm{P}_{2} \mathrm{O}_{5}$ 计). 随后分散到 不同浓度的 $\mathrm{Zn}\left(\mathrm{NO}_{3}\right)_{2}$ 溶液中, 充分搅拌, 浸渍 $4 \mathrm{~h}$, $110{ }^{\circ} \mathrm{C}$ 烘干并在 $550{ }^{\circ} \mathrm{C}$ 空气气氛下焙烧, 得到一系 列 $\mathrm{Zn}$ 负载量 $(1.5 \% 、 3 \%$ 和 $4.5 \%$ (质量分数)) 的 $\mathrm{Zn} / \mathrm{P} /$ ZSM-5. 最终得到的催化剂命名为 $a \% \mathrm{Zn} / \mathrm{P} / \mathrm{ZSM}-5$ $(R)$, 其中 $a \%$ 为 $\mathrm{Zn}$ 负载量, $R$ 为催化剂母体 $\mathrm{Si} / \mathrm{Al}$ 比.

氨吸附程序升温脱附 $\left(\mathrm{NH}_{3}\right.$-TPD) 在美国康塔公 司 ChemBET Pulsar 上进行. 将 $50 \mathrm{mg}$ 催化剂样品装 入测试装置内, 升温至 $600{ }^{\circ} \mathrm{C}$ 脱除催化剂上吸附的 气体物质, 降温至 $100^{\circ} \mathrm{C}$, 吸附氨气 $30 \mathrm{~min}$, 并使用 $\mathrm{He}$ 气吹出催化剂表面的物理吸附的氨气, 随后以 $10^{\circ} \mathrm{C} \cdot \mathrm{min}^{-1}$ 速率进行程序升温并在反应器出口位置 检测氨气的浓度. 最后, 通过标定单位体积氨气的信 号值来确定 ZSM-5 分子篎不同酸性位点的酸密度.

ZSM-5 催化剂中 B 酸和 Lewis 酸(L 酸)的分析 使用吡啶吸附的红外光谱法, 得到的酸量为半定量 结果. 仪器型号为 Nicolet Nexus 670, 工作区域为 $800-4000 \mathrm{~cm}^{-1}$, 分辨率 $4 \mathrm{~cm}^{-1}$. B 酸特征峰在 1545 $\mathrm{cm}^{-1}$ 附近, L 酸特征峰在 $1455 \mathrm{~cm}^{-1}$ 附近. 使用 1860 和 $1990 \mathrm{~cm}^{-1}$ 附近的分子篎特征峰归一化. ${ }^{26} \mathrm{~B}$ 酸密 度 $=1545 \mathrm{~cm}^{-1}$ 峰面积/(1860 $\mathrm{cm}^{-1}$ 特征峰面积 +1920 $\mathrm{cm}^{-1}$ 特征峰面积), $\mathrm{L}$ 酸量 $=1455 \mathrm{~cm}^{-1}$ 峰面积/(1860 $\mathrm{cm}^{-1}$ 特征峰面积 $+1920 \mathrm{~cm}^{-1}$ 特征峰面积). 取 $0.25 \mathrm{~g}$ 催化剂压成直径 $13 \mathrm{~mm}$ 的圆片, 置于真空池中, $450{ }^{\circ} \mathrm{C} 、 10^{-4} \mathrm{~Pa}$ 下脱气 $4 \mathrm{~h}$, 降温到 $100^{\circ} \mathrm{C}$, 吸附吡啶 分子 $1 \mathrm{~h}$, 升温到 $200^{\circ} \mathrm{C}$ 并抽真空到 $10^{-4} \mathrm{~Pa}$ 以下, $2 \mathrm{~h}$ 后谱图不再变化时采集谱图, 继续升温到 $350{ }^{\circ} \mathrm{C}$ 并 抽真空到 $10^{-4} \mathrm{~Pa}$ 以下, $2 \mathrm{~h}$ 后采集谱图.

催化剂的反应评价在微型反应装置中进行. 将 $0.2 \mathrm{~g}$ 催化剂置于直径 $10 \mathrm{~mm}$ 的固定床微反中, 将纯 甲醇通过预热器预热至 $200^{\circ} \mathrm{C}$, 通入反应器中在 $475{ }^{\circ} \mathrm{C}$ 下进行反应, 控制甲醇的质量空速为 0.79 $\mathrm{h}^{-1}$. 二甲苯或甲苯甲醇混合物进料时过程类似. 所 得气体与液体产品用气相色谱(日本岛津公司, GC2014)进行分析. 芳烃收率是指产物中的总芳烃 基于原料的烃基收率, 即不考虑甲醇中的水分子. 芳烃产品的归一化分布由苯、甲苯、二甲苯或三甲 苯的收率除以总芳烃收率后得到。

\section{3 结果与讨论}

\section{1 不同进料在 3\% Zn/P/ZSM-5(14)催化剂上的 转化反应}

积碳改性是 ZSM-5 常用的修饰手段. 一般认 为, 甲醇反应在催化剂上的积碳分布在外表面和孔 道内部, 导致酸性中心部分被覆盖. ${ }^{27}$ 红外和化学吸 附表明, 对不同强度的酸性位点, 催化剂积碳都会 引起酸密度的大量下降. 28

在 $3 \% \mathrm{Zn}$ 改性, 母体 $\mathrm{Si} / \mathrm{Al}$ 为 14 的 $3 \% \mathrm{Zn} / \mathrm{P} /$ ZSM-5(14)催化剂上进行甲醇转化反应, 随着反应 时间的延长, 催化剂上积碳量不断增加. 通过分析 积碳过程中各芳烃组分收率变化, 考察积碳对甲醇 制芳烃体系主次反应的影响.

图 1 为甲醇转化过程中芳烃收率. 如图 1 所示, 甲醇一次通过催化剂转化, 芳烃碳基收率约为 75\% 左右, 包括苯、甲苯、二甲苯、三甲苯和少量乙苯. 在 $142 \mathrm{~min}$ 的反应时间内, 总芳烃收率比较稳定, 说明 该催化剂芳构化的能力在积碳情况下没有明显降 低. 而另一方面, 各芳烃组分的收率随着积碳增加 却呈现规律性变化. 苯和甲苯的收率随反应进行逐 渐降低, 由 $15 \mathrm{~min}$ 时的 5.7\%和 21.2\%下降到 $142 \mathrm{~min}$ 时的 $2.1 \%$ 和 $9.5 \%$. 二甲苯的总收率基本不变, 同时 对、间、邻三个异构体的组成也保持在热力学平衡 组成附近, 即 $m$ (对位): $m$ (间位): $m$ (邻位) $=23: 53: 24$. 三甲苯的收率随反应进行增加, 由 $15 \mathrm{~min}$ 时的 $13.1 \%$ 增加到 $142 \mathrm{~min}$ 时的 $27.2 \%$. 苯和甲苯收率的 下降, 以及三甲苯收率的提高, 说明反应积碳后, 脱 烷基反应(减少芳环甲基侧链数目)部分被抑制, 而 烷基化反应(增加芳环甲基侧链数目)则大量发生. 为了验证这一点, 在类似的反应条件下 $\left(475{ }^{\circ} \mathrm{C} 、 0.1\right.$ $\mathrm{MPa} 、 2 \mathrm{~mL}$ /进料量), 换用不同进料进行转化反应, 得到原料转化率和产品分布结果见图 2 和图 3.

在甲苯和甲醇同时进料下, 甲醇除和甲苯发生 烷基化反应生成二甲苯外, 还可以单独发生芳构化

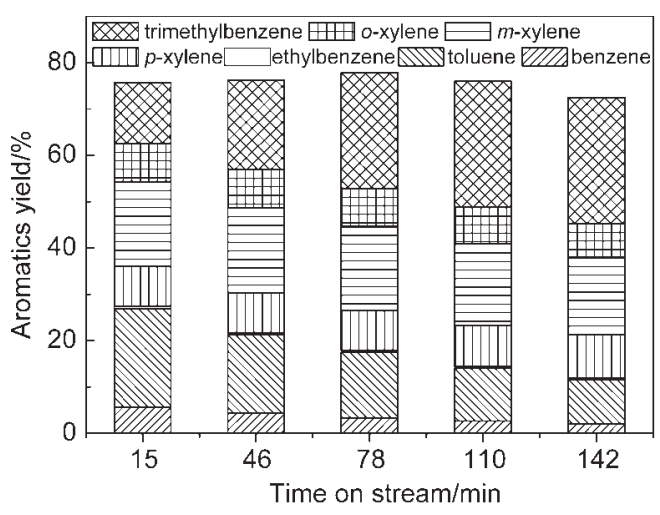

图 1 甲醇转化过程中芳烃收率

Fig.1 Aromatics yield in methanol conversion reaction $475^{\circ} \mathrm{C}, 0.1 \mathrm{MPa}, 0.79 \mathrm{~h}^{-1}, 3 \% \mathrm{Zn} / \mathrm{P} / \mathrm{ZSM}-5(14)$ 


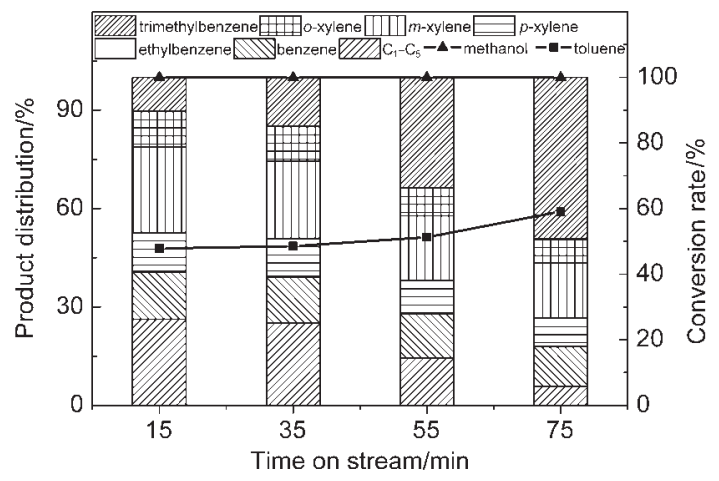

图 2 甲苯与甲醇同时进料时甲醇转化率和芳烃产品分布 Fig.2 Conversion rates and product distribution under toluene and methanol co-feeding

molar ratio of toluene to methanol $=1: 2,475^{\circ} \mathrm{C}, 0.1 \mathrm{MPa}, 0.83 \mathrm{~h}^{-1}$, $3 \% \mathrm{Zn} / \mathrm{P} / \mathrm{ZSM}-5(14)$

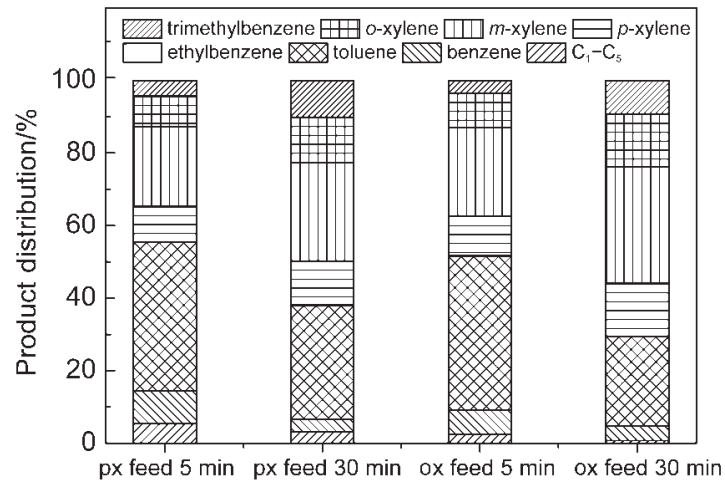

图 3 对二甲苯(px)或邻二甲苯(ox)转化的归一化产品分布

Fig.3 Normalized aromatics distribution in p-xylene (px) or $\boldsymbol{o}$-xylene (ox) conversion reaction

$475{ }^{\circ} \mathrm{C}, 0.1 \mathrm{MPa}, 0.43 \mathrm{~h}^{-1}, 3 \% \mathrm{Zn} / \mathrm{P} / \mathrm{ZSM}-5(14)$. Referred to the distribution of the mixture out of the reactor, including the unreacted px or ox.

反应, 生成 $\mathrm{C}_{6}-\mathrm{C}_{9}$ 芳烃以及 $\mathrm{C}_{1}-\mathrm{C}_{5}$ 烯烃和烷烃. 二甲 苯也可以与甲醇进一步烷基化生成三甲苯. 从图 2 中可以看到, 甲醇转化率基本保持在 $100 \%$, 而甲苯 转化率则逐渐增加, 由 $15 \mathrm{~min}$ 时的 $47.8 \%$ 增加到 75 $\min$ 时的 $59.1 \%$. 在产品分布中, 低碳烃类占比随反 应积碳增加逐渐下降, 由 $15 \mathrm{~min}$ 时的 $26.3 \%$ 降低到 $75 \mathrm{~min}$ 时的 $5.8 \%$. 其中以丙烷的减少最为明显, 由 $15 \mathrm{~min}$ 时的 $13.4 \%$ 降低到 $75 \mathrm{~min}$ 时的 $1.9 \%$. 在此进 料体系下低碳烃类, 尤其是丙烷的生成, 主要来源 于甲醇的芳构化反应. 该结果表明在大量甲苯存在 的情况下, 甲醇芳构化反应受到抑制. 并且随着反 应积碳的增加, 芳构化能力变差. 同时三甲苯的含 量随着反应进行不断增加, 说明二甲苯可以继续烷 基化生成三甲苯. 显然, 催化剂积碳量增加, 覆盖了 进行芳构化的强酸性位点, 剩余的弱酸性位点无芳
构化能力, 但对烷基化反应有利.

在对二甲苯或邻二甲苯进料的情形下, 主反应 包括: (1) 对二甲苯或邻二甲苯异构化得到热力学平 衡组成的混二甲苯; (2) 二甲苯发生脱烷基反应得到 甲苯和苯. 另外二甲苯还可能发生歧化和烷基化反 应, 得到甲苯和少量三甲苯. 从图 3 中可以看到, 不 管是对二甲苯进料还是邻二甲苯进料, 相比反应 5 $\min$ 时, 反应 $30 \mathrm{~min}$ 时二甲苯通过脱甲基得到的甲 苯大大减少. 说明催化剂少量积碳对脱烷基反应有 强烈的抑制作用.

甲苯与甲醇混合进料和二甲苯进料的实验证 明, 随着催化剂反应积碳, 脱烷基反应被抑制, 烷基 化反应则加快, 这和甲醇进料时的各芳烃收率的变 化是吻合的.

总之, 催化剂积碳量增加对芳构化、烷基化和 脱烷基反应的影响各不相同. 在甲醇进料时, 芳构 化能力不变, 而烷基化加快, 脱烷基反应被抑制, 这 也在甲苯甲醇混合进料和二甲苯进料的实验中得 到证实.

\section{2 不同母体 Si/AI 比和 $\mathrm{Zn}$ 负载量的 $\mathrm{Zn} / \mathrm{P} / \mathrm{ZSM}-5$ 催化剂上的甲醇转化反应}

反应积碳可以看做是对催化剂酸性的原位改 性，通过不同进料下的微反实验可以得到芳构化、 烷基化和脱烷基反应在积碳下的变化规律, 却难于 获得足够的表征结果和反应结果进行关联. 为此制 备了一系列不同母体 $\mathrm{Si} / \mathrm{Al}$ 比和 $\mathrm{Zn}$ 负载量的 $\mathrm{Zn} / \mathrm{P} /$ ZSM-5 催化剂, 在其上进行甲醇转化反应, 并通过 表征得到催化剂的酸强度、酸密度和酸性位点类型 等性质, 探讨催化剂酸性性质对芳构化、烷基化、脱 烷基和异构化反应的影响。

选用 $\mathrm{Si} / \mathrm{Al}$ 摩尔比为 $14 、 18.5 、 24.4$ 和 39.2 的 HZSM-5 催化剂, 负载 $3 \%$ 的 $\mathrm{P}$ 和 $3 \%$ 的 $\mathrm{Zn}$, 得到一系 列 3\% Zn/P/ZSM-5 催化剂. 利用 $\mathrm{NH}_{3}-\mathrm{TPD}$ 的方法分 析催化剂的酸强度和酸密度.

四个催化剂样品的 $\mathrm{NH}_{3}$-TPD 谱图如图 4 所示. 使用高斯分峰方法进行处理, 拟合数据和实验数据 能够较好地吻合. 谱图中峰的位置代表酸性位点的 强弱, 峰温越高, 酸性越强, 从左向右三个峰分别代 表弱酸、中强酸和强酸位点. 峰的面积代表酸密度 的大小, 可以利用氨气滴定做外标, 得到酸性位点 的酸密度.

如图 5 所示, 三种酸性位点的酸密度都随 $\mathrm{Si} / \mathrm{Al}$ 比的增加而减少, 但减小的幅度有所不同. 催化剂 

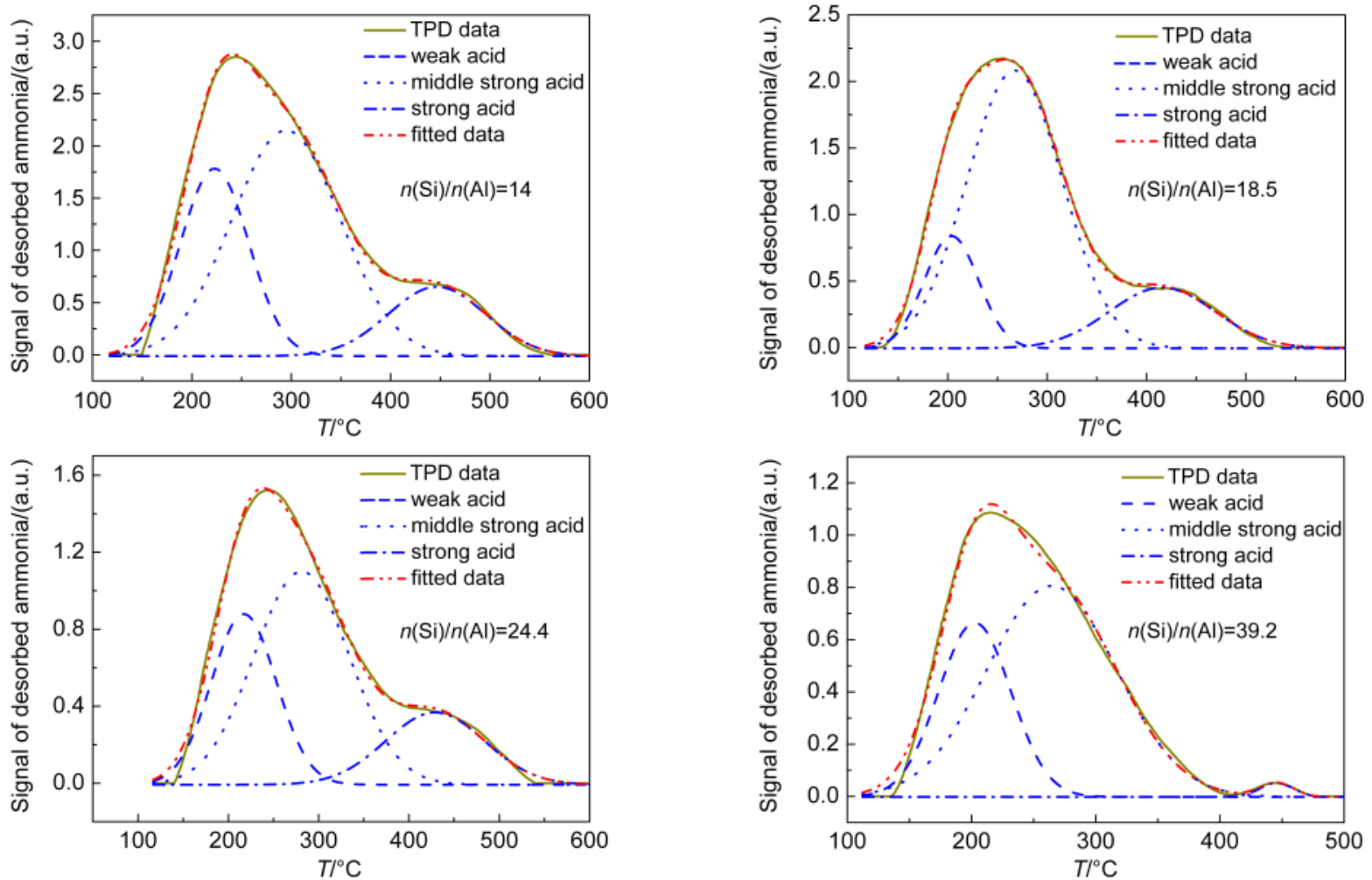

图 4 不同 $n(\mathrm{Si}) / n(\mathrm{Al})$ 比的 $\mathrm{Zn} / \mathrm{P} / \mathrm{ZSM}-5$ 的 $\mathrm{NH}_{3}$-TPD 谱图

Fig.4 $\mathrm{NH}_{3}$-TPD profiles of $\mathrm{Zn} / \mathrm{P} / \mathrm{ZSM}-5$ with different $n(\mathrm{Si}) / n(\mathrm{Al})$ ratios

$\mathrm{Si} / \mathrm{A} 1$ 比由 14 增加到 18.5 , 强酸、中强酸和弱酸的酸 密度大约按照相同的比例减少. $\mathrm{Si} / \mathrm{Al}$ 比由 18.5 增加 到 24.4, 弱酸酸密度下降放缓, 强酸和中强酸酸密度 仍然大量减少. $\mathrm{Si} / \mathrm{Al}$ 比由 24.4 增加到 39.2 , 中强酸酸 密度的下降也开始放缓, 而强酸酸密度下降趋势不 变. $\mathrm{Si} / \mathrm{Al}$ 比为 39.2 的样品强酸位点密度只有 0.006 $\mathrm{mmol} \cdot \mathrm{g}^{-1}$, 与其他三个样品存在数量级上的差距.

从图 6 可以看到, 催化剂 $\mathrm{Si} / \mathrm{Al}$ 比由 14 增加到 24.4, 催化剂强酸位点酸密度均匀下降, 但芳构化性 能变化并不大, 芳烃收率仅由 75.6\%下降到 73.9\%.

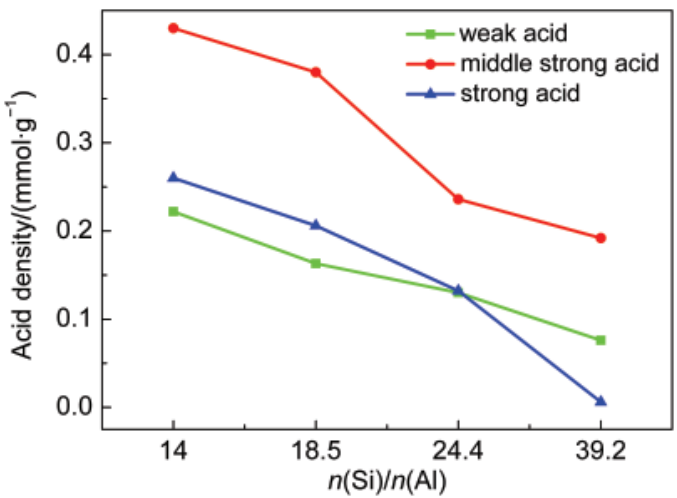

图 5 不同 $n(\mathrm{Si}) / n(\mathrm{Al})$ 比 $\mathrm{Zn} / \mathrm{P} / \mathrm{ZSM}-5$ 催化剂的酸密度

Fig.5 Acid density of Zn/P/ZSM-5 catalysts with different $n(\mathrm{Si}) / n(\mathrm{Al})$ ratios
而归一化的芳烃产品分布却发生了规律性的变化 (见图 7), 表现为苯和甲苯所占比例不断下降, 而三 甲苯所占比例增加, 二甲苯所占比例稍有增加. 这 与图 1 中各芳烃组分收率随积碳变化的规律是一 致. 这说明强酸位点酸密度的下降对脱烷基反应产 生了更大影响. 而当 $\mathrm{Si} / \mathrm{Al}$ 比由 24.4 增加到 39.2 时, 强酸位点的酸密度大幅度减少, 芳烃总收率降低到 $41.4 \%$, 对二甲苯在二甲苯中的选择性由热力学平

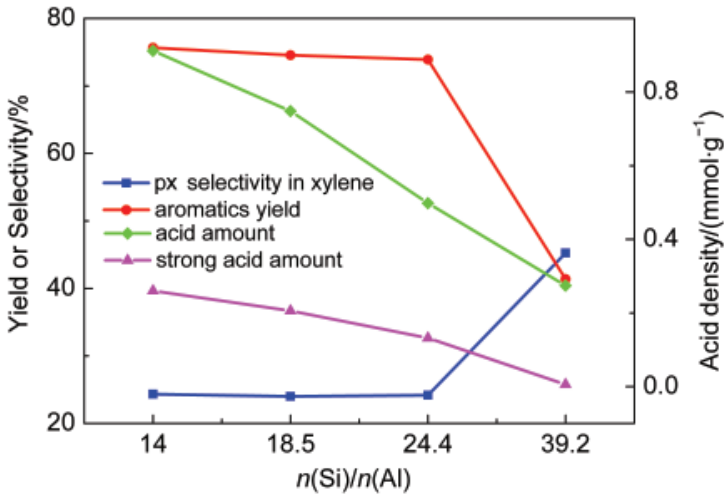

图 6 不同 $\mathrm{Si} / \mathrm{AI}$ 比的 $\mathrm{Zn} / \mathrm{P} / \mathrm{ZSM}-5$ 催化剂的酸密度及 甲醇反应结果

Fig.6 Acid amount and reaction results of $\mathrm{Zn} / \mathrm{P} / \mathrm{ZSM}-5$ catalysts with different $\boldsymbol{n}(\mathrm{Si}) / \boldsymbol{n}(\mathrm{Al})$ ratios

$475^{\circ} \mathrm{C}, 0.1 \mathrm{MPa}, 0.79 \mathrm{~h}^{-1}, 15 \mathrm{~min}$ after the reaction began. $n(\mathrm{Si}) / n(\mathrm{Al})$ ratios were determined by ICP analysis. 


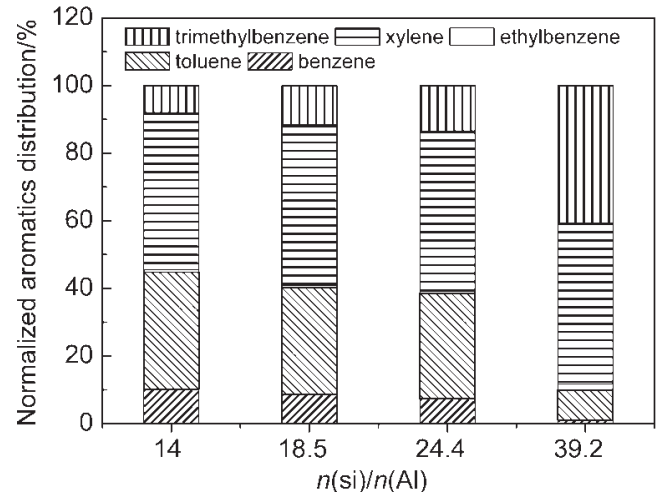

图 7 不同 $n(\mathrm{Si}) / \boldsymbol{n}(\mathrm{Al})$ 比催化剂归一化芳烃产品分布

Fig.7 Normalized aromatics distribution of Zn/P/ZSM-5 catalysts with different $\boldsymbol{n}(\mathrm{Si}) / \boldsymbol{n}(\mathrm{Al})$ ratios

$475{ }^{\circ} \mathrm{C}, 0.1 \mathrm{MPa}, 0.79 \mathrm{~h}^{-1}, 15 \mathrm{~min}$ after the reaction began

衡组成的 24\%增加到 $45 \%$. 二甲苯中对位异构体浓 度的提高表明, 从孔中扩散出的对二甲苯在外表面 的异构化反应得到部分抑制. 在强酸位点酸密度下 降到一定程度时, 芳构化反应和异构化反应都受到 抑制, 说明二者对催化剂酸强度和酸密度的要求相 近. 而在强酸位点酸密度超低的催化剂 $3 \% \mathrm{Zn} / \mathrm{P} /$ ZSM-5(39.2)上, 烷基化反应仍在进行, 得到大量的 二甲苯和三甲苯, 说明烷基化反应可在强度较低的 酸中心上进行.

另一方面, 为了考察酸性位点类型(B酸或 L 酸) 对甲醇芳构化过程主次反应的影响, 制备了一系列 不同 $\mathrm{Zn}$ 负载量及母体的 $\mathrm{Si} / \mathrm{Al}$ 比为 14 的 $\mathrm{Zn} / \mathrm{P} /$ ZSM-5(14). 吡啶吸附红外谱图如图 8 所示, 其中 $1455 \mathrm{~cm}^{-1}$ 处的峰为吡啶与 L 酸结合的特征峰, 1545 $\mathrm{cm}^{-1}$ 峰为吡啶与 $\mathrm{B}$ 酸结合的特征峰. 图中的实线是 $200{ }^{\circ} \mathrm{C}$ 下脱附吡啶后测量的红外谱图, 可以代表催 化剂强酸和弱酸的总和, 虚线是 $350^{\circ} \mathrm{C}$ 下脱附吡啶

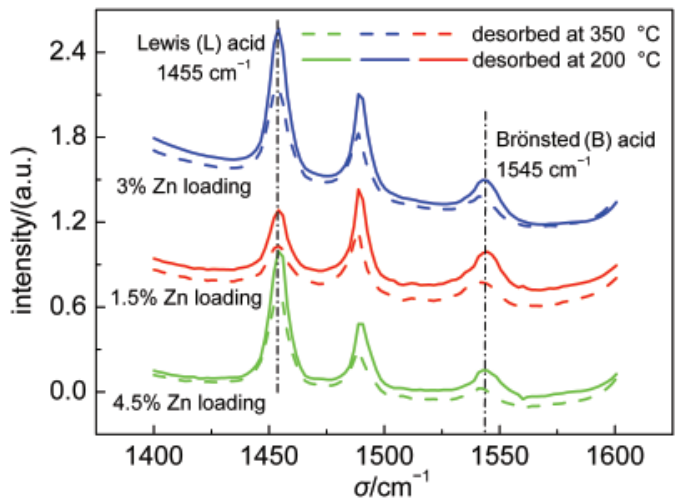

图 8 不同 $\mathrm{Zn}$ 负载量的 Zn/P/ZSM-5 催化剂吡啶 吸附红外谱图

Fig.8 Py-IR spectra of Zn/P/ZSM-5 catalysts with different $\mathrm{Zn}$ loadings

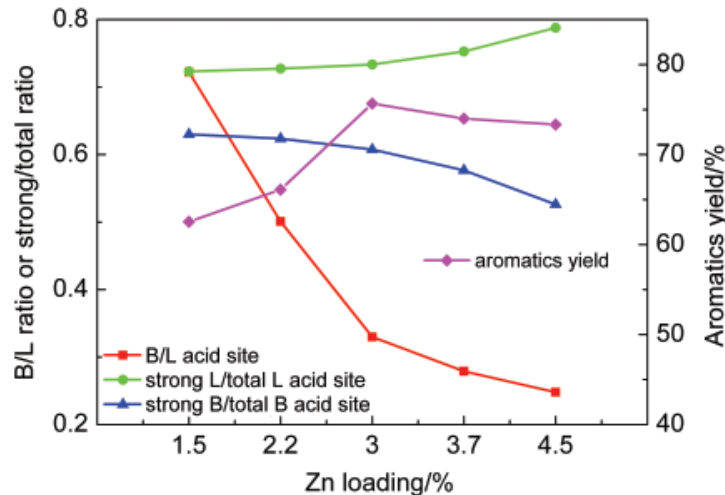

图 9 不同 $\mathrm{Zn}$ 负载量催化剂的酸性位点比例和 甲醇反应芳烃收率

Fig.9 Aromatics yield and acid site ratios of Zn/P/ZSM-5 catalysts with different $\mathrm{Zn}$ loadings

$475^{\circ} \mathrm{C}, 0.1 \mathrm{MPa}, 0.79 \mathrm{~h}^{-1}$, average result during the first hour.

后测量的红外谱图, 代表了催化剂的强 $\mathrm{B}$ 酸和强 $\mathrm{L}$ 酸的总和. 分别将 1455 和 $1545 \mathrm{~cm}^{-1}$ 峰面积进行归 一化处理, 代表 L 酸和 B 酸的酸量. 这种半定量的处 理方法适用于本文的讨论各种酸性位点的比例.

随着 $\mathrm{Zn}$ 负载量由 $1.5 \%$ 增加到 $4.5 \%, \mathrm{~L}$ 酸峰面 积逐渐增大, $\mathrm{B}$ 酸峰面积逐渐减小, $\mathrm{B}$ 酸/L 酸比下 降. 这是因为 $\mathrm{Zn}$ 的引入增加了 $\mathrm{L}$ 酸位点, 减少了 $\mathrm{B}$ 酸位点..$^{29,30}$ 如图 9 所示, 随着 $\mathrm{Zn}$ 负载量的增加和 $\mathrm{B}$ 酸/L酸比例的下降, 总芳烃收率呈现出先增大后减 少的趋势, 在 $\mathrm{Zn}$ 负载量为 $3 \%$ 时达到最大. 这是因为 芳构化是一个 $\mathrm{B}$ 酸与 $\mathrm{L}$ 酸协同作用的催化过程, ${ }^{31}$ 过 高的或过低的 $\mathrm{B} / \mathrm{L}$ 酸比例都对提高芳烃收率不利.

从图 9 中也看出, $\mathrm{Zn}$ 改性量对 $\mathrm{L}$ 酸和 $\mathrm{B}$ 酸的酸 强度有一定的影响. Zn 改性量由 $1.5 \%$ 增加到 $4.5 \%$, 强 Lewis 酸占总 Lewis 酸的比例由 $72 \%$ 增加到 $79 \%$. 而相反的是, 强 B 酸的总 B 酸的比例由 $63 \%$ 降低到

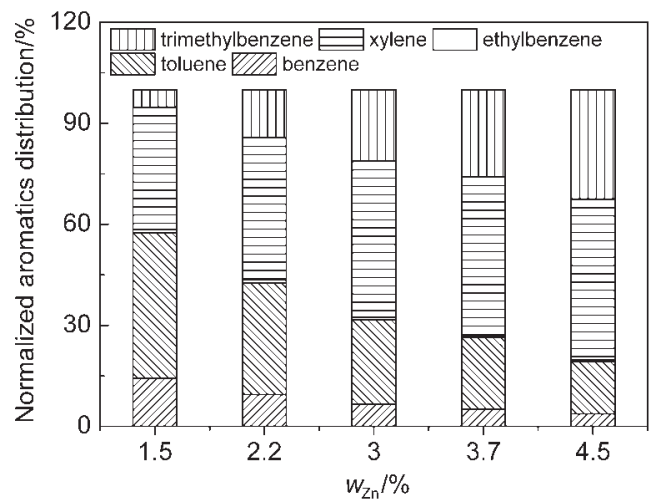

图 10 不同 Zn 负载量的 Zn/P/ZSM-5 催化剂所得 归一化芳烃产品分布

Fig.10 Normalized aromatics distribution of $\mathrm{Zn} / \mathrm{P} / \mathrm{ZSM}-5$ catalysts with different zinc loadings

$475^{\circ} \mathrm{C}, 0.1 \mathrm{MPa}, 0.79 \mathrm{~h}^{-1}$, average result during the first hour 

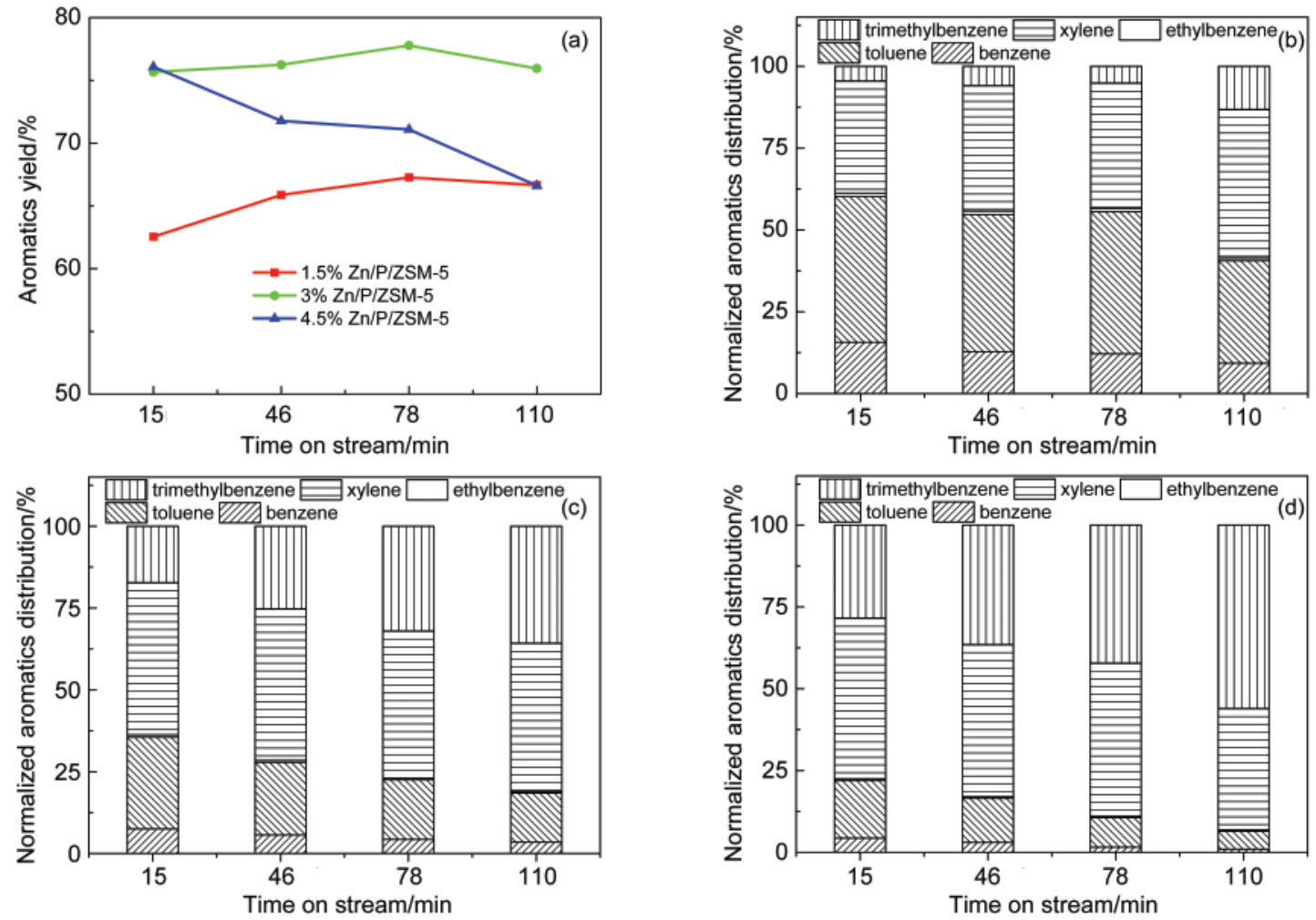

图 11 芳烃收率和产品分布随反应时间的变化

Fig.11 Aromatics yield and product distribution of Zn/P/ZSM-5 catalysts

(a) aromatic yield; (b) normalized aromatics distribution, $1.5 \% \mathrm{Zn} / \mathrm{P} / \mathrm{ZSM}-5(14)$; (c) normalized aromatics distribution, $3 \% \mathrm{Zn} / \mathrm{P} / \mathrm{ZSM}-5$ (14); (d) normalized aromatics distribution, $4.5 \% \mathrm{Zn} / \mathrm{P} / \mathrm{ZSM}-5$ (14)

$53 \% . \mathrm{B}$ 强酸比例的下降影响了芳烃归一化产品的 分布. 如图 10 所示, 随着 $\mathrm{Zn}$ 负载量的增加, 芳烃产 品中苯和甲苯占比持续减少, 三甲苯占比持续增 加. 这与图 1 和图 7 中芳烃分布的变化规律是类似 的, 说明了酸强度的降低不利于脱烷基反应, 但不 影响烷基化反应.

进一步考察 $1.5 \%$ 、3\%和 $4.5 \% \mathrm{Zn}$ 改性的催化剂 上芳烃收率和芳烃产品分布随反应积碳的变化. 如 图 11 所示, $1.5 \% \mathrm{Zn}$ 改性的催化剂随反应时间延长 芳烃收率不断增加, 这可能是因 $1.5 \% \mathrm{Zn}$ 改性的催 化剂本身具有过高的 B 酸/L 酸比例, 反应积碳使 B 酸/L 酸比例下降, 有利于芳烃收率的提高. 而 $4.5 \%$ $\mathrm{Zn}$ 改性的催化剂本身具有过低的 $\mathrm{B}$ 酸/L 酸比例, 表 现出了恰好相反的规律, 随反应时间延长, 芳烃收 率不断下降. 3\% Zn 改性的催化剂芳烃收率基本不 变, 与图 1 相比, 这两个实验的催化剂改性方法相同 但批次不同, 结果是类似的. 而在芳烃产品分布上, 不管 $\mathrm{Zn}$ 改性量的多少, 苯和甲苯所占比例都随反应 时间延长而下降, 说明在不同程度上脱烷基反应都 得到了抑制. $1.5 \% \mathrm{Zn}$ 改性的催化剂上二甲苯和三
甲苯占比都随反应时间延长而增加; $3 \% \mathrm{Zn}$ 改性的 催化剂上二甲苯占比随反应时间延长基本不变, 三 甲苯占比随反应时间延长增加; $4.5 \% \mathrm{Zn}$ 改性的催 化剂上二甲苯占比随反应时间延长下降, 三甲苯所 占比例随反应时间延长增加. 这说明催化剂强酸位 点酸密度不同, 烷基化反应进行的程度也不同. 强 酸位点数目越少, 越容易发生彻底的烷基化生成三 甲苯. 强酸位点数目过高, 烷基化反应不充分, 二甲 苯收率不高. $3 \% \mathrm{Zn}$ 改性的催化剂强酸位点数目合 适, 因此具有较高且稳定的二甲苯收率.

选用母体 $\mathrm{Si} / \mathrm{Al}$ 比为 $14 、 3 \% \mathrm{Zn}$ 负载量的 $\mathrm{Zn} / \mathrm{P} /$ ZSM-5 进行甲醇转化, 在 $100 \%$ 的转化率下能够得 到 75\% 的初始芳烃收率, 经过两个多小时的反应积 碳后, 芳构化能力基本不变.二甲苯收率高达 $35 \%$, 且不随积碳增加而发生变化. 该催化剂具有良好的 工业应用前景.

\section{4 结 论}

(1) 制备的 Zn/P/ZSM-5 系列催化剂具有良好的 芳构化性能. 其中母体 $\mathrm{Si} / \mathrm{Al}$ 比为 $14 、 3 \% \mathrm{Zn}$ 改性的 
催化剂具有较高的强酸位点酸密度和合适的 $\mathrm{B}$ 酸/ $\mathrm{L}$ 酸比例, 甲醇转化的芳烃收率高达 $75 \%$.

（2）随着催化剂反应积碳, 芳构化能力基本不 变, 而脱烷基反应受到抑制, 烷基化反应在严重积 碳后仍然进行.

(3) 不同 $\mathrm{Si} / \mathrm{Al}$ 比和 $\mathrm{Zn}$ 负载量催化剂上的甲醇 转化反应表明, 芳构化、脱烷基和烷基化反应对强 酸位点酸密度要求不同. 随着强酸位点酸密度下 降. 首先受到抑制的是脱烷基反应，其次是芳构化 反应, 而烷基化反应在强酸密度很低的情况下仍能 进行, 并且将生成大量三甲苯. 控制强酸位点酸密 度可以有效地控制芳烃产品分布.

（4）芳构化和异构化反应对强酸位点酸密度的 要求相似, 因此, 在消除酸性位点来提高对位选择 性的同时往往会带来芳烃收率的下降. 考虑到异构 化反应基本在外表面进行, 为了尽可能减少对芳构 化反应的影响, 选择性消除外表面酸性位点是抑制 异构化反应的一个思路.

\section{References}

(1) Olsbye, U.; Svelle, S.; Bjorgen, M.; Beato, P.; Janssens, T. V. W.; Joensen, F.; Bordiga, S.; Lillerud, K. P. Angew. Chem. Int. Edit. 2012, 51, 2. doi: 10.1002/anie.201107584

(2) Keil, F. J. Microporous Mesoporous Mat. 1999, 29, 49. doi: 10.1016/S1387-1811(98)00320-5

(3) Stocker, M. Microporous Mesoporous Mat. 1999, 29, 3. doi: 10.1016/S1387-1811(98)00319-9

(4) Zaidi, H. A.; Pant, K. K. Catal. Today 2004, 96, 155. doi: 10.1016/j.cattod.2004.06.123

(5) Freeman, D.; Wells, R. P. K.; Hutchings, G. J. J. Catal. 2002, 205, 358. doi: 10.1006/jcat.2001.3446

(6) Freeman, D.; Wells, R. P. K.; Hutchings, G. J. Chem. Commun. 2001, 1754 .

(7) Ni, Y. M.; Peng, W. Y.; Sun, A. M.; Mao, W. L.; Hu, J. L.; Li, T.; Li, G. X. J. Ind. Eng. Chem. 2010, 16, 503. doi: 10.1016/j. jiec.2010.03.011

(8) Lopez-Sanchez, J. A.; Conte, M.; Landon, P.; Zhou, W.; Bartley, J. K.; Taylor, S. H.; Carley, A. F.; Kiely, C. J.; Khalid, K.; Hutchings, G. J. Catal. Lett. 2012, 142, 1049. doi: 10.1007/ s10562-012-0869-2

(9) Tian, T.; Qian, W. Z.; Sun, Y. J.; Cui, Y.; Lu, Y. Y.; Wei, F. Modern Chemical Industry 2009, 29, 55. [田 涛, 寒伟中, 孙 玉建, 崔 宇, 卢俨俨, 魏 飞. 现代化工, 2009, 29, 55.]

(10) Tian, T.; Qian, W. Z.; Tang, X. P.; Yun, S.; Wei, F. Acta
Phys. -Chim. Sin. 2010, 26, 3305. [田 涛, 寒伟中, 汤效平, 恽 松, 魏飞. 物理化学学报, 2010, 26, 3305.] doi: 10.3866/ PKU.WHXB20101228

(11) Wang, J. Y.; Li, W. H.; Hu, J. X. Journal of Fuel Chemistry and Technology 2009, 37, 607. [王金英, 李文怀, 胡津仙. 燃料化 学学报, 2009, 37, 607.]

(12) Kecskemeti, A.; Barthos, R.; Solymosi, F. J. Catal. 2008, 258, 111. doi: 10.1016/j.jcat.2008.06.003

(13) Choudhary, V. R.; Panjala, D.; Banerjee, S. Appl. Catal. A 2002, 231, 243. doi: 10.1016/S0926-860X(02)00061-3

(14) Inoue, Y.; Nakashiro, K.; Ono, Y. Microporous Mat. 1995, 4, 379. doi: 10.1016/0927-6513(95)00020-A

(15) Bjørgen, M.; Svelle, S.; Joensen, F.; Nerlov, J.; Kolboe, S.; Bonino, F.; Palumbo, L.; Bordiga, S.; Olsbye, U. J. Catal. 2007, 249, 195. doi: 10.1016/j.jcat.2007.04.006

(16) Adebajo, M. O.; Long, M. A. Catal. Commun. 2003, 4, 71. doi: 10.1016/S1566-7367(02)00259-5

(17) Lukyanov, D. B.; Gnep, N. S.; Guisnet, M. R. Ind. Eng. Chem. Res. 1995, 34, 516. doi: 10.1021/ie00041a012

(18) Olson, D. H.; Kokotailo, G. T.; Lawton, S. L.; Meler, W. M. J. Phys. Chem. 1981, 85, 2238. doi: 10.1021/j150615a020

(19) Joshi, Y. V.; Thomson, K. T. J. Phys. Chem. C 2008, 112, 12825. doi: 10.1021/jp712071k

(20) Olson, D. H.; Haag, W. O. ACS Symp. Ser. 1984, 248, 275. doi: 10.1021/symposium

(21) Mirth, G.; Cejka, J.; Lercher, J. A. J. Catal. 1993, 139, 24. doi: 10.1006/jcat.1993.1003

(22) Guisnet, M.; Gnep, N. S.; Morin, S. Microporous Mesoporous Mat. 2000, 35-36, 47.

(23) Ivanova, I. I.; Corma, A. J. Phys. Chem. B 1997, 101, 547. doi: $10.1021 / \mathrm{jp} 961468 \mathrm{k}$

(24) Tsai, T.; Liu, S.; Wang, I. Appl. Catal. A 1999, 181, 355. doi: 10.1016/S0926-860X(98)00396-2

(25) Serra, J. M.; Guillon, E.; Corma, A. J. Catal. 2004, 227, 459. doi: 10.1016/j.jcat.2004.08.006

(26) Zheng, S. R.; Heydenrych, H. R.; Jentys, A.; Lercher, J. A. J. Phys. Chem. B 2002, 106, 9552. doi: 10.1021/jp014091d

(27) Bibby, D. M.; Howe, R. F.; Mclellan, G. D. Appl. Catal. A 1992, 93, 1. doi: 10.1016/0926-860X(92)80291-J

(28) Benito, P. L.; Gayubo, A. G.; Aguayo, A. T.; Olazar, M.; Bilbao, J. Ind. Eng. Chem. Res. 1996, 35, 3991. doi: 10.1021/ie950462z

(29) Biscardi, J. A.; Meitzner, G. D.; Iglesia, E. J. Catal. 1998, 179 , 192. doi: 10.1006/jcat.1998.2177

(30) El-Malki, E. M.; Van Santen, R. A.; Sachtler, W. M. H. J. Phys. Chem. B 1999, 103, 4611. doi: 10.1021/jp9901161

(31) Bhan, A.; Delgass, W. N. Catal. Rev. 2008, 50, 19. doi: 10.1080/ 01614940701804745 